\title{
Classification of brain tumours by CT-image Walsh spectra
}

\author{
M. Wolf, S.Ziegengeist, M. Michalik, F. Bornholdt, S. Michalik, and B. Meffert \\ Klinik und Poliklinik für Neurologie und Psychiatrie des Bereiches Medizin (Charité) und Sektion Elektronik der Humboldt-Universităt \\ Berlin, Federal Republic of Germany
}

Received: 17 January 1990

Summary. Besides clinical and anamnestic data, image information from CT-image data in the tumour border was used for classification of brain tumours. If the image regions are properly selected a classification rate of $85 \%$ is obtained with a hierarchic classifier, although our study is based on only 139 patients.

Key words: Brain tumours - Pattern recognition - Computed tomography

Direct decision from digital image information (computer vision) is a complex task. Therefore the evaluation of images is often based on visually assessed features and on data measured with operator interaction by standard measuring software.

We devised a system for the differentiation of the 7 most common supratentorial brain tumors. It uses pixel data from windows located in the tumour border, where tumour specific structures are expected. An alternative set of features, consisting of patient data and image features such as distances and densities measured with standard CT image evaluation software was also tested. First both feature sets were evaluated separately, then they were combined, because both obviously contained complementary information (Fig.1). For classification and data analysis linear discriminant analysis was used.

The major problem in using pixel data for classification is an effective algorithm, that reduces the large amount of image data, but maintains useful information. For this the two-dimensional Walsh-transform was used. Walsh- and Fourier-transforms are able to derive stable statistical features of large-area patterns of "noisy" appearance, while there is evidence that human vision (in analogy to the detection of large-area bar patterns) analyses only small areas and is less efficient [4]. We also had CT's of 14 histologically proven astrocytomas evaluated by 7 experienced neurologists. Less than $60 \%$ of them were classified correctly and only $26 \%$ with the correct grade.

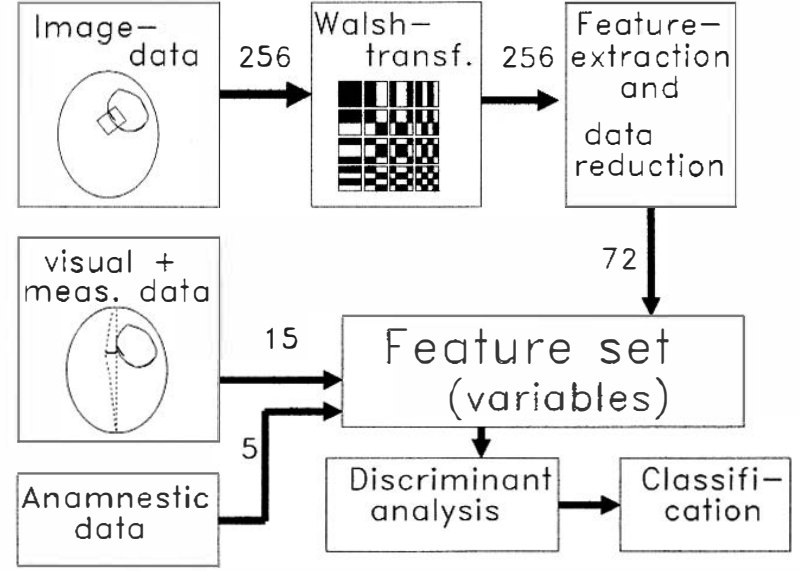

Fig. 1. Information used for classification

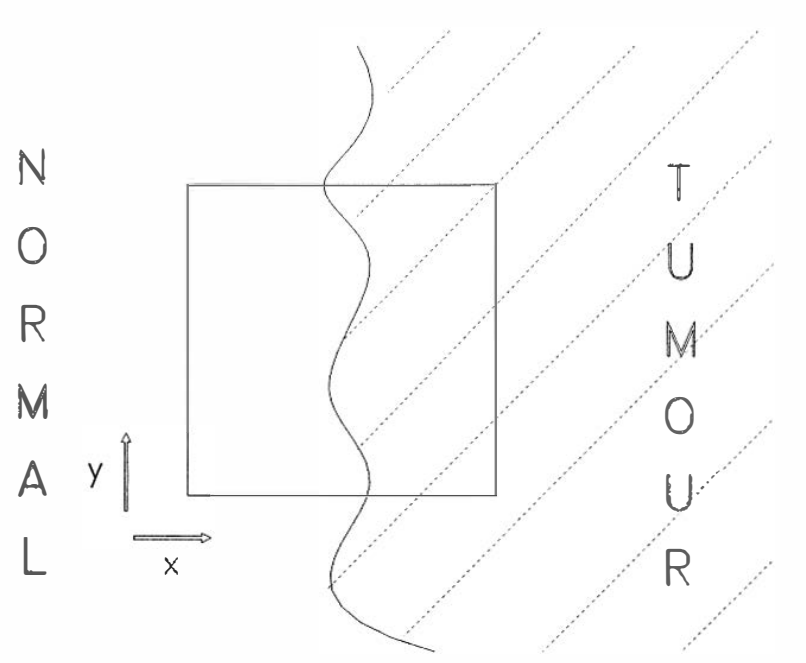

Fig.2. Standard position of the window for Walsh-transform centered at the tumour border, where the Walsh-coefficient $\mathrm{C}(0,1)$ is a maximum. The tumour is at the right-hand side 


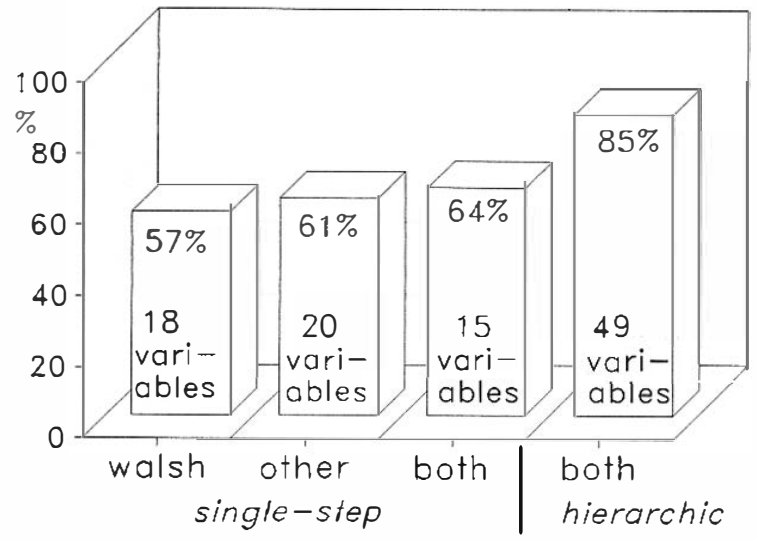

Fig.3. Rates of true classifications and the optimum number of features selected for decision by discriminant analysis for the two sets of features, their combination and for hierarchic classification

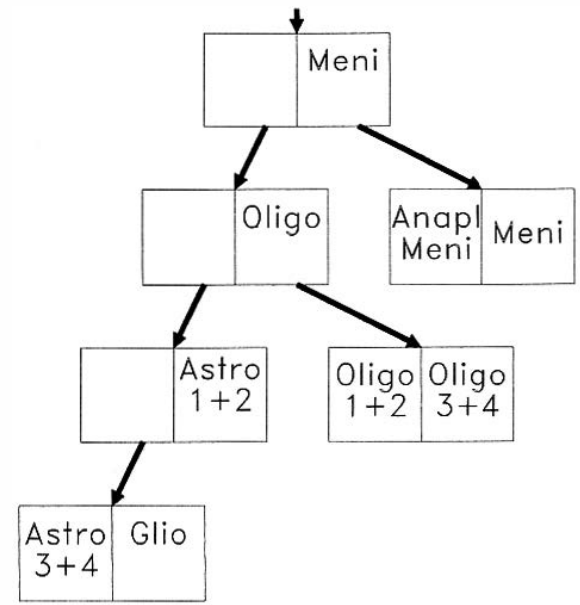

Fig.4. Structure of hierarchic classifier

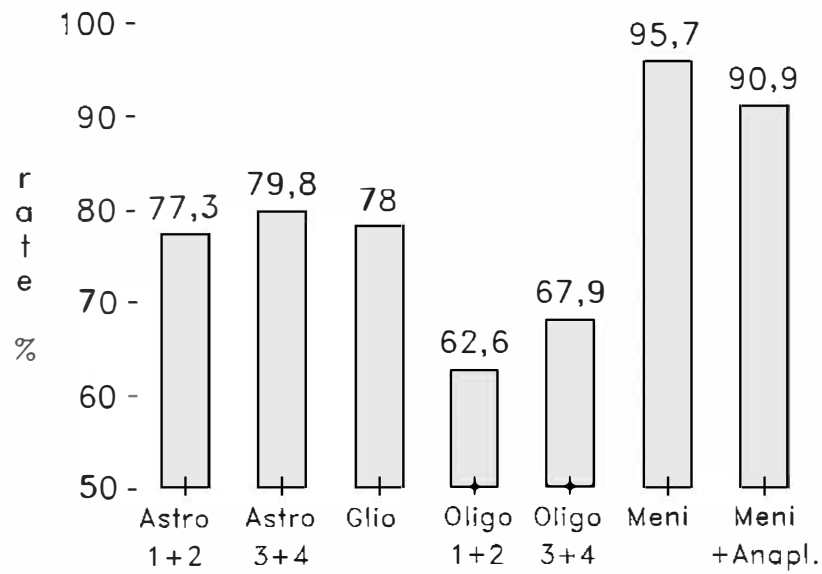

Fig. 5. Rates of correct classifications for the hierarchic system

\section{Method and technical parameters}

Our study is based on a relatively small sample of 248 scans from 139 patients with histologically proven diagnosis (Table 1). Main limiting factor for the number of patients is the availability of images in digital form.
Table 1. Composition of the test sample

\begin{tabular}{llcc}
\hline Class & & Patients & Scans \\
\hline 1 Astrocytoma & grade 1 +2 & 31 & 51 \\
2 Astrocytoma & grade 3+4 & 23 & 39 \\
3 Glioblastoma & & 36 & 68 \\
4 Oligodendroglioma & grade 1 +2 & 10 & 14 \\
5 Oligodendroglioma & grade 3+4 & 6 & 11 \\
8 Meningeoma & non-anaplastic & 28 & 54 \\
9 Meningeoma & anaplastic & 5 & 11 \\
\hline Total & & 139 & 248 \\
\hline
\end{tabular}

Table 2. The alternative set of features

\begin{tabular}{|c|c|}
\hline Measured image features & Visually assessed image features \\
\hline 1. Average tumour density & 12. Tumour density (hypo-/ \\
\hline 2. Average tumour density with & iso-/hyperdens) \\
\hline contrast medium & 13. Calcifications \\
\hline 3. Standard dev of density in the & 14. Lecalisation \\
\hline tumour & 15. Ventricle compression \\
\hline 4. Standard dev. of density with & 16. Hydrocephalus \\
\hline contrast medium & 17. Spread across center line \\
\hline 5. Displacement of center line & 18. Size of oedema \\
\hline $\begin{array}{l}\text { 6. Ratio of displacement of cen- } \\
\text { ter line to diameter of tumour }\end{array}$ & $\begin{array}{l}\text { 19. Pattern of contrast medium } \\
\text { (qualitative description) } \\
\text { 20. Delineation of tumour with }\end{array}$ \\
\hline Anamnestic features & contrast medium. \\
\hline 7. Age & \\
\hline 8. Sex & \\
\hline 9. Duration of anamnesis & \\
\hline 10. first significant symptom & \\
\hline 11. Progression & \\
\hline
\end{tabular}

CT-images were taken with a Siemens Somatom, at $125 \mathrm{kV}, 230 \mathrm{mAs}, 8 \mathrm{~mm}$ slice thickness, and a measuring time of 10 s per slice. Generally, scans were made with and without contrast medium for each patient (Visotrast 290; $1 \mathrm{ml}$ per kilogram body weight).

From each scan we selected up to 4 windows of $16 \times 16$ pixels, centered at the tumour border.

From their Walsh-spectrum a shift-invariant set of features with 72 coefficients is derived and averaged from 4 windows per scan. The altemative set of data is formed by 20 features (Table 2).

\section{Results and conclusions}

Both feature sets were investigated with discriminant analysis. The prospective rate of true classifications is estimated with the so-called Pi-method. $1 / 10$ of the available cases is evaluated by a classifier derived from the rest of the cases, and this process is repeated 10 times [1].

The discriminant analysis program selects a subset of variables, for which the classification rate becomes a maximum (Fig. 3). For the combined set of features the number of selected parameters was less than for both sets alone. This indicates, that both feature sets either contain redundant information, or that linear singlestep discrimination does not use the information efficiently. 
Therefore we calculated an hierarchic classifier for the combined set of data (Fig. 4). The calculation of 6 binary classifiers is laborious, but yields a much betterrate of estimated true classifications $-85 \%$. Different features are selected at each stage, and the total number of used features is much higher ( 49 of 92 ) than in the single-step procedure (15 of 92 ).

Figure 5 shows, that Meningeomas are well detected, while the separation of oligodendrogliomas is more difficult. The rate of correct classifications therefore varies with the composition of the investigated sample of patients. For a distribution of the tumour types derived from the literature it is $85 \%$, for our sample $82 \%$ were calculated. Despite the small number of patients this result is comparable to that of other authors [2,3], although a practically applicable system would have to cope with more than only 7 alternative diagnoses [3].

While the statistical calculations are time consuming, the Walsh-transforms and the final decision are accomplished within less than $10 \mathrm{~s}$ on the PDP-11 computer of the Evaluskop. The results justify the development of an improved program with pull-down menus, operated with the pen of the systems console.

\section{References}

1. Ahrens H, Läuter J (1981) Mehrdimensionale Varianzanalyse. Akademie-Verlag, Berlin

2. Iglesias JR, Aruffo C, Esparza J, Trempeneau B, Kazner E (1987) An expert system for the diagnosis of brain tumours. In: Lemke HU, Rhodes ML, Jaffee CC, Felix R (eds) Computer assisted radiology (CAR 87). Springer, Berlin, p 397

3. Teather D, Teather BA, Wills KM, du Boulay GH, Plummer D, Isherwood I, Gholkar A (1988) Evaluation of computer advisor in the interpretation of CT images of the head. Neuroradiology 30 : $511-517$

4. Wolf M (1980) Signal-to-noise ratio and the detection of detail in non-white noise. Photogr Sci Eng 24: 99-103

Dr. M.Wolf

Klinik und Poliklinik fü

Neurologie der Charité

Schumannstrasse 20/21

1040 Berlin

Federal Republic of Germany 